\title{
Complex Variable and Regularization Methods of Inversion of the Laplace Transform
}

\author{
By D. D. Ang, John Lund, and Frank Stenger
}

\begin{abstract}
In this paper three methods are derived for approximating $f$, given its Laplace transform $g$ on $(0, \infty)$, i.e., $\int_{0}^{\infty} f(t) \exp (-s t) d t=g(s)$. Assuming that $g \in$ $L^{2}(0, \infty)$, the first method is based on a Sinc-like rational approximation of $g$, the second on a Sinc solution of the integral equation $\int_{0}^{\infty} f(t) \exp (-s t) d t=g(s)$ via standard regularization, and the third method is based on first converting $\int_{0}^{\infty} f(t) \exp (-s t) d t=g(s)$ to a convolution integral over $\mathbf{R}$, and then finding a Sinc approximation to $f$ via the application of a special regularization procedure to solve the Fourier transform problem. We also obtain bounds on the error of approximation, which depend on both the method of approximation and the regularization parameter.
\end{abstract}

\section{Introduction and Summary.}

1.1. In the present paper we develop three new methods for carrying out the numerical inversion of the Laplace transform. That is, if $\mathbb{R}^{+}$denotes the interval $(0, \infty)$, we obtain accurate approximations to $f$ defined on $\mathbb{R}^{+}$by

$$
\mathscr{L} f=g
$$

where $g$ is given on $\mathbb{R}^{+}$. In $(1.1), \mathscr{L} f$ is defined by

$$
(\mathscr{L} f)(s)=\int_{0}^{\infty} e^{-s t} f(t) d t
$$

The Laplace transform occurs frequently in the applications of mathematics [2], $[3],[35]$, especially those involving the solution of partial differential equations. Here it is used far less frequently as a tool of approximation than the Fourier transform, in part, because there are no universal methods of inverting the Laplace transform, but mainly, because in methods such as separation of variables, $g$ is known only on $\mathbb{R}^{+}$, and the use of the Bromwich inversion formula [43, p. 67]

$$
f(t)=\frac{1}{2 \pi i} \int_{c-i \infty}^{c+i \infty} e^{s t} g(s) d s
$$

is therefore not feasible. The fact that the methods of this paper require only a knowledge of $g$ on $\mathbf{R}^{+}$thus circumvents this difficulty. The lack of universal methods for inverting the Laplace transform stems from the fact that the space of functions $f$ for which $\mathscr{L} f$ exists is simply too big. We immediately restrict this space by assuming that $f \in L^{2}\left(\mathbb{R}^{+}\right)$, which implies [14] that $g \in L^{2}\left(\mathbb{R}^{+}\right)$. In applications it

Received April 20, 1987; revised June 20, 1988 and October 11, 1988.

1980 Mathematics Subject Classification (1985 Revision). Primary 44A10; Secondary 65R10, $45 \mathrm{~L} 10$.

Key words and phrases. Laplace transform, inversion. 
is generally possible to achieve this criterion via elementary manipulations, which we shall discuss below. An excellent summary of other methods for inverting the Laplace transform is contained in [10]. Furthermore, the methods summarized in [10] are tested on several functions. While the tests in [10] are interesting, the criteria of testing do not restrict the space of functions, and it is thus possible to write down test functions for which any one of the methods does extremely well, while all of the others fail miserably. A variety of other methods are discussed in [4], [16], [17], [22], [23], [30], [44], [45], [46].

The main feature of the three methods of this paper are the following:

(a) The methods require that we be able to evaluate $g$ on $\mathbb{R}^{+}$.

(b) Two of the three methods allow for the presence of noisy data.

(c) If for some positive number $\alpha$ the function $f$ satisfies

$$
f(t)= \begin{cases}O\left(t^{\alpha}\right) & \text { as } t \rightarrow 0 \\ O\left(t^{-\alpha}\right) & \text { as } t \rightarrow \infty\end{cases}
$$

and if $f$ is analytic on $(0, \infty)$, then all of the methods work well. In particular, it is not necessary to know the explicit nature of the singularity of $f(t)$ at $t=0$ or at $t=\infty$. Indeed, the second and third methods do not work if $f(t)$ does not approach zero at $t=0$ and at $t=\infty$. In Subsection 1.3 below we discuss simple procedures for altering $g$ so that $f$ satisfies (1.4).

(d) The computer algorithms for achieving inversion are both very simple, and very short.

(e) Of the three methods, the first applies to the largest class of functions, and hence it is the slowest; the second, which is the only one requiring the solution of a linear system of equations, applies to a larger class of functions than the third, while the third applies to the smallest class of functions (see (c) above) and is the fastest of the three.

(f) All three of the methods involve regularization. This regularization is implicit in the first method, while in the second and third methods a regularization parameter must be selected. Indeed, the second and third methods fail if the regularization parameter is taken to be zero.

1.2. In this subsection we shall summarize the results of this paper. The proofs are deferred to later sections.

Let us define the usual $p$-norm for integrable functions on an interval $(a, b)$ by

$$
\|g\|_{p}=\left(\int_{a}^{b}|g(x)|^{p} d x\right)^{1 / p}, \quad 1 \leq p<\infty .
$$

In most cases we shall use the $p=2$ norm, and on such occasions we shall at times omit the subscript $p$, i.e., $\|\cdot\|=\|\cdot\|_{2}$.

Let $\mathbb{C}$ denote the complex numbers, and let us define regions $\Omega_{+}, D_{d}, S_{d}$ and $B_{d}$, where $d>0$, by

$$
\begin{aligned}
\Omega_{+} & =\{z \in \mathbb{C}: \operatorname{Re} z>0\} \\
D_{d} & =\{z \in \mathbb{C}:|\operatorname{Im} z|<d\} \\
S_{d} & =\{z \in \mathbb{C}:|\arg z|<d ; \\
B_{d} & =\{z \in \mathbb{C}:|\arg \sinh z|<d\} .
\end{aligned}
$$


Here, $\Omega_{+}$denotes the right half plane, and illustrations of the regions $D_{d}, S_{d}$ and $B_{d}$ can be found in [39, pp. 177 and 186].

Method I. In this method we assume the absence of noise in the data $g$. This method was discovered first, and its does not use a regularization parameter. When $g$ is replaced by an accurate rational function approximation derived in [40], it is possible to use the calculus of residues to evaluate explicitly the Bromwich integral (1.3). Although Method I is convergent, it does not converge as rapidly as Methods II and III in the case when Methods II and III also work. However, its explicit nature offers the possibility of further developmental work, and it may therefore be possible to develop a very efficient method from it. The method is summarized in the following theorem.

THEOREM 1.1. Let $g$ defined as in Eq. (1.1) be analytic on $\Omega_{+}$, and let $g$ satisfy the relation*

$$
\|g\|=\lim _{\varepsilon \rightarrow 0^{+}}\left(\int_{\mathbf{R}}|g(\varepsilon+i u)|^{2} d u\right)^{1 / 2}<\infty .
$$

If $\delta(g, h, t)$ is defined for positive $h$ and $t$ by

$$
\delta(g, h, t)=f(t)-\left(\frac{h}{2 \pi}\right)^{2} e^{\pi^{2} / h} \sum_{m, n=-\infty}^{\infty}\left(-e^{h}\right)^{m+n} \frac{\exp \left(-t e^{m h}\right) g\left(e^{n h}\right)}{e^{m h}+e^{n h}},
$$

then for every $c>0$,

$$
\int_{\mathbf{R}^{+}} e^{-2 c t}|\delta(g, h, t)|^{2} d t \rightarrow 0
$$

as $h \rightarrow 0$.

Method II. The direct numerical solution of the first-kind integral equation $\mathscr{L} f=g$ is not possible [41], no matter how accurately we interpolate $g$. However, standard integral equation regularization greatly enhances the solution of this equation. Instead of $\mathscr{L} f=g$, we solve

$$
\beta f_{\beta}+\mathscr{L}^{*} \mathscr{L} f_{\beta}=\mathscr{L}^{*} g
$$

where $\beta$ is a positive number, and where $\mathscr{L}^{*}=\mathscr{L}$, since $\mathscr{L}$ is selfadjoint [14]. Written in full, (1.10) takes the form

$$
\beta f_{\beta}(t)+\int_{0}^{\infty} \frac{f_{\beta}(u)}{u+t} d u=\int_{0}^{\infty} e^{-s t} g(s) d s
$$

The role of the regularization parameter $\beta$, whose selection is discussed below, is especially important if the "data" $g$ is contaminated with noise, in which case $\mathscr{L} f=g$ may not have a solution, whereas a solution of $(1.11)$ still exists. The following result recommends a choice of $\beta$ in the presence of noise.

ThEOREM 2.1. Let $\mathscr{L} f=g_{0}$, where $g_{0} \in L^{2}\left(\mathbb{R}^{+}\right)$, and let $f=\mathscr{L} u$ for some $u \in L^{2}\left(\mathbf{R}^{+}\right)$, where $\|u\|=\left(\int_{\mathbf{R}^{+}}|u(t)|^{2} d t\right)^{1 / 2} \leq E$. If $\left\|g-g_{0}\right\| \leq \varepsilon$, then for $\beta=\varepsilon$, the solution $f_{\beta}$ of (1.10) satisfies the inequality

$$
\left\|f_{\beta}-f\right\| \leq(1+E) \varepsilon^{1 / 2} / 2 .
$$

\footnotetext{
${ }^{*}$ This assumption is in fact a consequence of $f \in L^{2}\left(\mathbf{R}^{+}\right)$.
} 
We next describe numerical procedures for solving (1.11). To this end, we make the following assumptions.

Assumption 2.2. Let $\mathscr{L} f=g$, and let $f$ be analytic and bounded in $D$, where $D$ denotes one of the regions $S_{d}$ or $B_{d}$ defined in (1.6). If $f$ is analytic and bounded in $S_{d}$, we define $\phi, z_{k}$, and $w_{k}$ by

$$
\phi(t)=\log t, \quad z_{k}=e^{k h}, \quad w_{k}=e^{k h} .
$$

In this case, we assume furthermore that for $t \in \mathbb{R}^{+}$and for some positive number $\alpha$,

$$
f(t)= \begin{cases}O\left(t^{\alpha}\right), & t \rightarrow 0 \\ O\left(t^{-\alpha}\right), & t \rightarrow \infty .\end{cases}
$$

If $f$ is analytic and bounded in $B_{d}$ we define $\phi, z_{n}$ and $w_{k}$ by

$$
\begin{aligned}
& \phi(t)=\log \sinh (t), \\
& z_{k}=\log \left[e^{k h}+\left(1+e^{2 k h}\right)^{1 / 2}\right], \quad w_{k}=\left(1+e^{-2 k h}\right)^{-1 / 2},
\end{aligned}
$$

and we assume that for some $\alpha>0$ and $t$ on $\mathbb{R}^{+}, f$ satisfies the relations

$$
f(t)= \begin{cases}O\left(t^{\alpha}\right), & t \rightarrow 0 \\ O\left(e^{-\alpha t}\right), & t \rightarrow \infty\end{cases}
$$

In Eqs. (1.13) and (1.15), let $h$ be selected by the formula

$$
h=\left(\frac{\pi d}{\alpha N}\right)^{1 / 2} \text {. }
$$

THEOREM 2.3. Let Assumption 2.2 be satisfied, and let the sequence of numbers $\left\{f_{m}\right\}_{-N}^{N}$ be determined by the solution of the linear system of equations

$$
\beta f_{m}+h \sum_{k=-N}^{N} \frac{w_{k} f_{k}}{z_{m}+z_{k}}=h \sum_{k=-N}^{N} w_{k} g\left(z_{k}\right) e^{-z_{m} z_{k}} .
$$

Let $f_{\beta, N}$ be defined on $\mathbb{R}^{+}$by

$$
f_{\beta, N}(t)=\sum_{m=-N}^{N} f_{m} S(m, h) \circ \phi(t)
$$

where $S(m, h)(u)=(-1)^{m}(h / \pi)(u-m h)^{-1} \sin [(\pi / h) u]$. Then there exists a constant $C$ which is independent of $N$, such that

$$
\sup _{t \in \mathbf{R}^{+}}\left|f_{\beta}(t)-f_{\beta, N}(t)\right| \leq C N^{1 / 2} e^{-(\pi d \alpha N)^{1 / 2}} .
$$

We remark that in the statement of Theorem 2.3 we have neglected the role of the condition number of the matrix in the linear system (1.18). The role of this condition number is further discussed in Subsection 1.4.

Method III. In this method we first convert Eqs. (1.1)-(1.2) into a convolution over $\mathbf{R}$, thus enabling the application of accurate Sinc quadrature [39] to get an accurate Fourier transform. A special type of regularization is thus employed to get a Sinc approximation of $f$. Eqs. (1.1)-(1.2) take the form

$$
\int_{0}^{\infty} e^{-s t} f(t) d t \cong g(s), \quad 0<s<\infty
$$




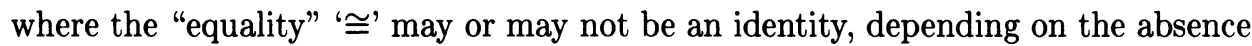
or presence of noise. By making the substitutions

$$
\begin{aligned}
& t=e^{u}, \quad s=e^{-v}, \\
& G(u)=e^{-u} g\left(e^{-u}\right), \quad F(u)=f\left(e^{u}\right), \\
& H(u)=\exp \left[-e^{-u}\right] e^{-u},
\end{aligned}
$$

we are able to reduce (1.21) to the convolution integral equation

$$
G(v) \cong \int_{\mathbf{R}} H(v-u) F(u) d u .
$$

Assuming the Fourier transform $\hat{G}, \hat{H}$ and $\hat{F}$ of $G, H$, and $F$ to exist, where for example

$$
\hat{G}(x)=\int_{\mathbf{R}} e^{i x u} G(u) d u,
$$

one has the approximation

$$
\hat{F} \cong \hat{G} / \hat{H}
$$

Assumption 3.1. Assume that for some $\hat{G}$ and $\hat{G}_{0}$ in $L^{2}(\mathbb{R})$, we have $\hat{F}$ in $L^{2}(\mathbb{R})$, such that

$$
\hat{F}=\hat{G}_{0} / \hat{H}
$$

and

$$
\left\|\hat{G}-\hat{G}_{0}\right\| \leq \varepsilon, \quad\|\hat{F}\| \leq E_{0} .
$$

For given constant $\beta>0$, let $\hat{F}_{\beta}$ be defined on $\mathbb{R}$ by

$$
\hat{F}_{\beta}(x)=\frac{\overline{\hat{H}}(x) \hat{G}(x)}{\beta+|\hat{H}(x)|^{2}} .
$$

THEOREM 3.2. If Assumption 3.1 is satisfied, then the choice $\beta=\varepsilon / E_{0}$ yields

$$
\left\|\hat{F}_{\beta}-\hat{F}\right\| \leq 2 E_{0} .
$$

THEOREM 3.3. Let the conditions of Theorem 3.2 be satisfied. In addition, assume that for some $s>0$,

$$
\int_{\mathbf{R}} x^{2 s}|\hat{F}(x)|^{2} d x \leq E_{s}<\infty .
$$

Furthermore, let $\alpha_{s}$ and $x_{s}>0$ be defined by

$$
\alpha_{s}=\sup _{x \in R}\left|x^{s} \hat{H}(x)\right|, \quad\left|\hat{H}\left(x_{s}\right)\right|^{2}=\frac{4 E_{0} \varepsilon}{\alpha_{s} E_{0}+E_{s}} x_{s}^{2 s} .
$$

Then

$$
\left\|\hat{F}_{\beta}-\hat{F}\right\| \leq \frac{2^{1 / 2}\left[\alpha_{s} E_{0}+E_{s}\right]^{1 / 2}}{x_{s}^{2 s}}
$$

where

$$
x_{s}=\frac{1}{\pi} \log \left(\frac{1}{\varepsilon}\right)[1+o(1)], \quad \varepsilon \rightarrow 0 .
$$


THEOREM 3.4. Let Assumption 3.1 hold, and in addition, let us assume that for some $d>0$,

$$
\int_{\mathbf{R}} \pi|x| e^{2 d|x|}\left|\hat{F}_{\beta}(x)-\hat{F}(x)\right|^{2} d x \leq E_{d}<\infty .
$$

Then, with $\beta=\varepsilon / E_{0}$,

$$
\left\|\hat{F}_{\beta}-\hat{F}\right\| \leq \frac{2 E_{d}(\pi+2 d)^{1 / 2}}{\pi \log \left[E_{d} / 2 E_{0} \varepsilon\right]}\left(\frac{.2 E_{0} \varepsilon}{E_{d}}\right)^{d /(\pi+2 d)} .
$$

Next, let us discuss the numerical approximations of $\hat{F}_{\beta}$ and $f_{\beta}$.

Assumption 3.5. Let $N$ denote a positive integer, let $h>0, \beta>0$, and let $\overline{\hat{H}}_{h}$, $\hat{G}_{h}$ and $\hat{F}_{\beta, h}$ be defined by

$$
\begin{aligned}
& \overline{\hat{H}}_{h}(x)=h \sum_{m=-N}^{N} e^{m h} \exp \left(-e^{m h}\right) e^{i m h x}, \\
& \hat{G}_{h}(x)=h \sum_{m=-N}^{N} e^{-m h} g\left(e^{-m h}\right) e^{i m h x}
\end{aligned}
$$

and

$$
\hat{F}_{\beta, h}(x)=\frac{\overline{\hat{H}}_{h}(x) \hat{G}_{h}(x)}{\beta+\pi x / \sinh (\pi x)} .
$$

Set $x_{j}=\pi j /(N h), j=-N, \ldots, N, F_{j}=F_{\beta, h}\left(x_{j}\right)$, and define $c_{k}(k=-N, \ldots, N)$ and $f_{\beta, h}^{(N)}$ by

$$
\begin{aligned}
& c_{k}=\frac{1}{2 N h}\left\{\frac{(-1)^{k}}{2}\left[F_{-N}+F_{N}\right]+\sum_{j=-(N-1)}^{N-1} F_{j} e^{-i k j / N}\right\} \\
& f_{\beta, h}^{(N)}(t)=\sum_{k=-N}^{N} c_{k} S(k, h) \circ \log t .
\end{aligned}
$$

THEOREM 3.6. Let $\mathscr{L} f=g$, and let $f$ satisfy Assumption 2.2 corresponding to the region $S_{d}$. Let $f_{\beta}$ be defined on $\mathrm{R}^{+}$by

$$
f_{\beta}(t)=\frac{1}{2 \pi} \int_{\mathbf{R}} \exp \{-i x \log t\} \hat{F}_{\beta}(x) d x,
$$

where $\hat{F}_{\beta}$ is defined by (1.28). If Assumption 3.5 holds, then there exists a constant $C$, which is independent of $N$ and $\varepsilon$, such that

$$
\sup _{0<t<\infty}\left|f_{\beta}(t)-f_{\beta, h}^{(N)}(t)\right|<C N^{1 / 2} e^{-(\pi d \alpha N)^{1 / 2}} .
$$

COROLlary 3.7. Let $\alpha$ be a positive constant, and let $\hat{F}$ defined by $\hat{F}=\hat{G} / \hat{H}$ be analytic and bounded in $S_{\alpha}$, where $S_{\alpha}$ is defined as $S_{d}$ was by Eq. (1.6). Let $\hat{F}_{\beta}$ be defined by (1.28), let $\|\hat{F}\|<E$, and let (1.34) be satisfied. Then there exists a constant $C$, which is independent of $N$ and $\varepsilon$, such that (1.40) holds.

1.3. There is no simple numerical method which accurately inverts every Laplace transform. Indeed, this conclusion was arrived at experimentally in [10], where several methods of inversion were tested on a variety of Laplace transforms. 
While the methods of the present paper also cannot be used to invert every Laplace transform, we shall nevertheless describe here a simple procedure for altering the original given $g$ to a new function $\tilde{g}$, where $\tilde{g}$ is such that we can guarantee its accurate inversion.

Best results obtain for all three methods if the desired function $f$ approaches zero as rapidly as possible, as $t \rightarrow 0$ and as $t \rightarrow \infty$. On the other hand, all methods work poorly or not at all, if $f(t)$ does not approach zero at least at an algebraic rate as $t \rightarrow \infty$.

Towards the estimation of the asymptotic behavior of $f(t)$ as $t \rightarrow 0$, let us choose two positive variables, $\sigma$ and $\tau$, such that

$$
\sigma \tau=1
$$

Let $L$ be any function with the property that for every fixed $\lambda>0$,

$$
\frac{L(\lambda x)}{L(x)} \rightarrow 1, \quad x \rightarrow \infty .
$$

For example, $L(x)=\log x$, or $L(x)=\log \left[a^{2}+\left(x^{2}+b^{2}\right)^{\alpha}\right], \alpha>0$, are such functions.

THEOREM 1.3.1 [13, p. 44]. Let $L$ be defined as above and $g=\mathscr{L} f$. Then each of the relations

$$
g(\sigma) \sim \sigma^{-\rho} L\left(\frac{1}{\sigma}\right), \quad \sigma \rightarrow 0(\text { resp. } \sigma \rightarrow \infty)
$$

and

$$
\int_{0}^{\tau} f(u) d u \sim \frac{1}{\Gamma(\rho+1)} \tau^{\rho} L(\tau), \quad \tau \rightarrow \infty(\text { resp. } \tau \rightarrow 0)
$$

implies the other.

Theorem 1.3.1 may be readily applied in practice to achieve the asymptotic rates (1.4) $\left(O\left(t^{\alpha}\right)\right.$ and $O\left(t^{-\alpha}\right)$, resp. as $\left.t \rightarrow 0, \infty\right)$ which we need in order for each of the methods of this paper to work effectively. To this end, the following functions and their corresponding transforms are basic:

TABLE 1.3 .1

Basic Transforms

\begin{tabular}{|c|c|}
\hline$f(t)$ & $g(s)$ \\
\hline$t^{\alpha-1} e^{-\beta t}$ & $\frac{\Gamma(\alpha)}{(s+\beta)^{\alpha}}(\alpha>0)$ \\
$t^{\alpha-1} \log t$ & $\Gamma(\alpha) s^{-\alpha}[\psi(\alpha)-\log s]$ \\
& $\left(\psi(\alpha)=\Gamma^{\prime}(\alpha) / \Gamma(\alpha)\right)$ \\
\hline
\end{tabular}


For example, given the transform of $f(t)=Y_{0}(t)$,

$$
g(s)=-\frac{2}{\pi}\left(s^{2}+1\right)^{-1 / 2} \log \left[s+\left(s^{2}+1\right)^{1 / 2}\right],
$$

which satisfies

$$
g(s)=-\frac{2}{\pi}\left[\frac{\log s}{s}+\frac{\log 2}{s}+O\left(\frac{\log s}{s^{2}}\right)\right], \quad s \rightarrow \infty,
$$

we have, by Theorem 1.3.1,

$$
f(t) \sim \frac{2}{\pi}[\log t+\text { const }], \quad t \rightarrow 0 .
$$

Hence, in view of Theorem 1.3.1 and Table 1.3.1, it follows that $g_{1}$ defined by

$$
g_{1}(s)=g(s)+\frac{2}{\pi}\left[\frac{\log (s+1)}{s+1}+\frac{\log 2}{s+1}\right]
$$

is the transform of $f_{1}(t)$, which has the satisfactory behavior

$$
f_{1}(t)=O(t \log t), \quad t \rightarrow 0 .
$$

On the other hand, Theorem 1.3.1 tells us nothing about the behavior of $f_{1}(t)$ as $t \rightarrow \infty$. Suitable asymptotic behavior as $t \rightarrow \infty$ may be achieved by inverting $g_{1}(s+a), a>0$, rather than $g_{1}(s)$. This would then enable us to accurately recover $e^{-a t} f_{1}(t)$, rather than $f_{1}(t)$ (compare [27]).

1.4. In the presence of noise, the choice of the regularization parameter $\beta$, which was used in Methods II and III, was probably an optimal one. However, we cannot take $\beta$ to be zero when carrying out the numerical computation of $f$ in Methods II and III, even in the absence of noise.

A generalization of the regularization procedure of Method III, which would be applicable to arbitrary convolution integral equations of the first kind, would be to replace $(1.28)$ by $\hat{F}_{\beta}(x)=\overline{\hat{H}}(x) \hat{G}(x) /\left[\beta \hat{W}(x)+|\hat{H}(x)|^{2}\right]$, where $\hat{W}(x)$ is some arbitrary positive and nondecreasing function of $|x|$. This generalization has advantages over the one recommended in [9], even for $\hat{W}(x)=|x|^{2 p}$. The procedure [9] recommends replacing our (1.28) by

$$
\hat{F}_{\beta}(x)=\{\hat{G}(x) / \hat{H}(x)\}\left\{|\hat{H}(x)|^{2} /\left[\beta|x|^{2 p}+|\hat{H}(x)|^{2}\right]\right\}
$$

however, this replacement does not guard against the denominator function $\hat{H}(x)$ becoming arbitrarily small or possibly (in a different problem) vanishing for finite $x$.

An acceptable value of $\beta$ to be used in the numerical evaluation of $f$ may be determined in the case when we know an accurate bound on the error of best Sinc approximation of $f$ on $\mathbb{R}^{+}$. Such an error may be considered to result from solving exactly a Laplace transform inversion problem (1.1) with a different $g$. Hence, by Theorems 2.1 and (e.g.) 3.4, it follows that our choice of $\beta$ is of the order of the error of the best Sinc approximation of $f$ on $\mathbb{R}^{+}$. The final error in the computed $f$ will thus be of the order of $\beta^{1 / 2}$.

On the other hand, if an accurate bound on the error of best Sinc approximation of $f$ on $\mathbf{R}^{+}$is not known, we are not unduly handicapped, since the error in the final approximation of $f$ obtained by either Methods II or III is a very slowly varying function of $\beta$ for $\beta$ larger than the best value, say $\beta_{b}$. The computed results become 
$L^{\infty}$ error $\times 10^{2}$

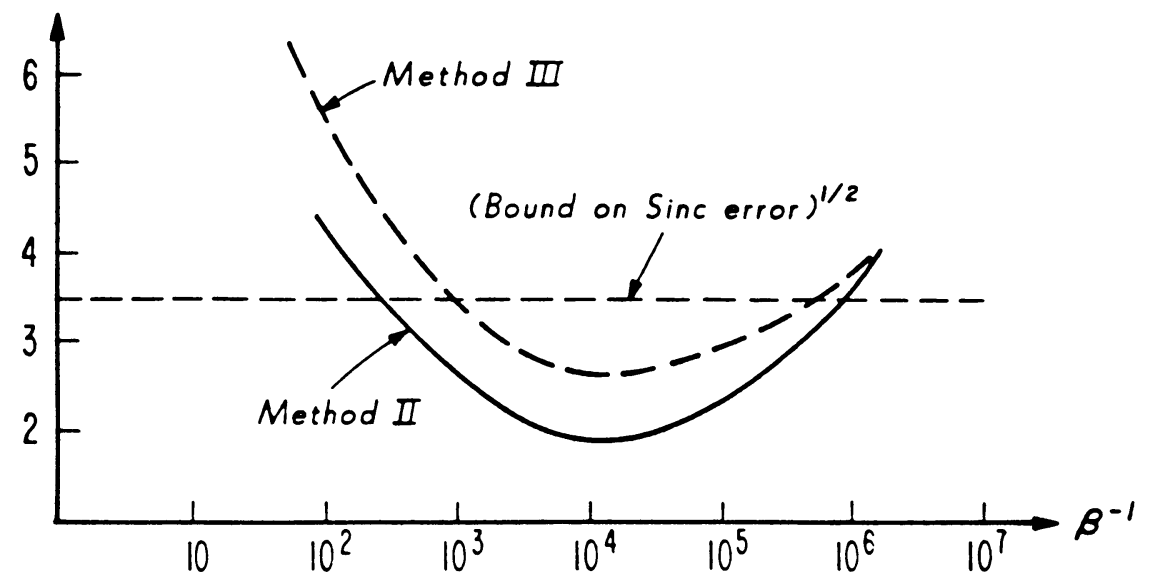

FIGURE 1.4.1. $L^{\infty}$ error versus $1 / \beta$

Exact $f(t)=t^{3 / 2} e^{-t} \ln t, N=11$

$L^{\infty}$ error $\times 10^{3}$

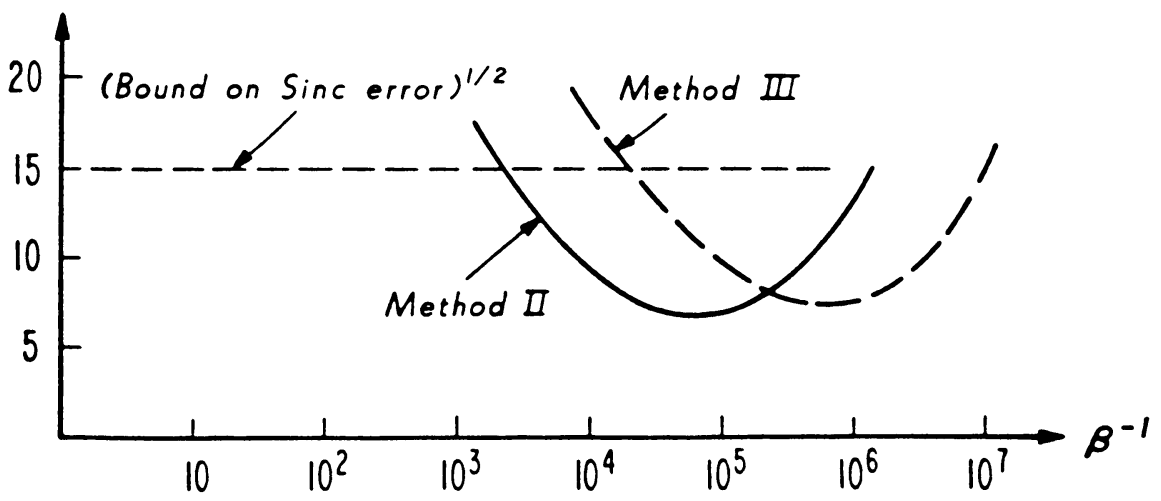

FIGURE 1.4.2. $L^{\infty}$ error versus $1 / \beta$

Exact $f(t)=t^{3 / 2} e^{-t} \ln t, N=21$

quite erratic in the region $0<\beta<\beta_{b}$. Two typical graphs illustrating the relation of the $L^{\infty}\left(\mathbf{R}^{+}\right)$error in the computed $f$ as a function of $1 / \beta$ are illustrated in Figure 1.4.1 (for the case of $N=11$ ) and 1.4.2 (for the case of $N=21$ ).

We remark also that the computations were done in IEEE double-precision floating-point format, on an HP 9000 (Model 320). For small $N$ (e.g., $N=5$ ) the computed solution remained bounded, even at $\beta=0$. As $N$ was increased, however, the errors in the $\beta=0$ solutions became increasingly large.

Let us recall that the implementation of Method II requires the numerical solution of a system of linear algebraic equations (see Eqs. (1.18)), whereas Method III does not. The condition number of the linear system (1.18) did not appear to affect the results of our computations in the ranges of $N$ and $\beta$ in Figures 1.4.1 and 1.4.2, possibly since the computations were carried out in double precision. Preliminary tests indicate, however, that in single precision, the results of Method II are not reliable for $\beta$ small (of the order of $10^{-6}$ or smaller). 
1.5. Given $g=\mathscr{L} f$, where $f \in L^{2}\left(\mathbb{R}^{+}\right)$, the inversion formula (1.3) (the Bromwich integral) is valid for every positive number $c$. Every method of approximating Fourier transforms applies also to the evaluation of the Bromwich formula (1.3). The most direct procedures involve the application of the trapezoidal rule to approximate the integral in (1.3), and many papers have been written on this method [6], [7], [8], [10], [18], [21], [38]. While this procedure is straightforward, complex values of $g$ are required, and many evaluation points are required, since the integrand usually approaches zero very slowly as $s \rightarrow c \pm i \infty$ along the line $\operatorname{Re} s=c$. Other ingenious methods that are useful for approximating Fourier transforms, such as via Möbius inversion [15], or by using splines [24], may at times produce a rapidly convergent integral.

Another approach is to assume a certain type of (Galerkin) approximation for $f$, with unknown coefficients, to take the Laplace transform, and to minimize in some way, the difference between $g(s)$ and the approximate transform [1], [5], [11], [12], [27], [29], [30], [34], [36]. These approximation schemes are sometimes also combined with the Post inversion formula [31],

$$
f(t)=\lim _{n \rightarrow \infty} \frac{(-1)^{n}}{n !}\left(\frac{n}{t}\right)^{n+1} g^{(n)}\left(\frac{n}{t}\right)
$$

(see [33], [37], [40]).

Error bounds for approximate inversion of Laplace transforms have been previously obtained in [27], where $f$ was itself assumed to be a Laplace transform, and where $f$ was approximated by decreasing exponentials, and in [26] for more general methods also, of inversion of Laplace transforms. The bounds in [26] apply also to our methods, although our conditions of applicability are more easily checked. Our error analyses, as well as the numerical stability of our methods, are closely tied to the selection of the best Tikhonov parameter $\beta$. This parameter $\beta$ allows for the presence of noise in $g$, and moreover, Methods II and III of our paper would not work at all if $\beta$ were taken to be zero, even in the absence of noise in $g$.

Various numerical algorithms based on Tikhonov regularization [40] have been devised for solving the matrix systems that arise in the approximate solution of Fredholm integral equations of the first kind [20], [42], and which have been applied to obtain approximate inversion of Laplace transforms [20], [25]. While it is possible to achieve good numerical results in this way, the methods of the present paper are designed specifically for inversion of Laplace transforms, and they are therefore more efficient.

2. Proof of Theorem 1.1. In this section we carry out a detailed proof of Theorem 1.1.

We achieve the interpolation of $g$ on $\mathbf{R}^{+}$by means of the function

$$
\phi(z, h)=\frac{z-1}{z+1} \prod_{j=1}^{\infty} \frac{\left(z-e^{j h}\right)\left(\frac{1}{z}-e^{j h}\right)}{\left(z+e^{j h}\right)\left(\frac{1}{z}+e^{j h}\right)},
$$

where $h>0$ and $z$ is an arbitrary complex number. The function $\phi(z, h)$ has zeros at the same points $\left(e^{ \pm j h}\right)$ as the function $\sin [(\pi / h) \log z]$ which was used in [39] to achieve Sinc interpolation on $\mathbf{R}^{+}$; indeed, as was shown in [40], the interpolation properties on $\mathbf{R}^{+}$of the interpolant which we shall derive via (2.1) are similar to 
those of the Sinc interpolation formulas obtainable via $\sin [(\pi / h) \log z]$. The function $\phi(z, h)$ has a unique advantage over $\sin [(\pi / h) \log z]$ in that it also has poles on the negative real line, and it is this property which makes it possible to get an explicit approximation for $f(t)$ via the Bromwich integral (1.3).

The function $\phi$ is in fact an elliptic function. In order to see this, we introduce here the following standard notation for elliptic functions:

$$
\begin{aligned}
& k \in(0,1), \\
& \begin{array}{l}
\omega=\omega(u ; k)=\int_{0}^{u}\left[\left(1-t^{2}\right)\left(1-k^{2} t^{2}\right)\right]^{-1 / 2} d t \\
\Leftrightarrow u=\operatorname{sn}(\omega ; k),
\end{array} \\
& K=K(k)=\omega(1 ; k), \\
& k^{\prime}=\left(1-k^{2}\right)^{1 / 2}, \\
& K^{\prime}=K\left(k^{\prime}\right), \\
& q=\exp \left(-\pi K^{\prime} / K\right), \\
& q^{\prime}=\exp \left(-\pi K / K^{\prime}\right) .
\end{aligned}
$$

Setting

$$
e^{2 i v}=t, \quad q=e^{-h / 2}
$$

in $[28$, Eqs. 16.37.1, 16.37.2] and using [28, Eq. 16.36.3], we conclude that

$$
\phi(z, h)=\left(k^{\prime}\right)^{1 / 2} \operatorname{sn}\left[\frac{K}{\pi} \log z ; k^{\prime}\right] .
$$

Properties of $\phi$ and some estimates on the parameters $K, k^{\prime}$ as functions of $h$ are recorded in the following two lemmas.

LEMMA 2.1. Let $\phi$ be defined by (2.1), where $z \in \mathbb{C}$ and $h>0$. Then (a) For all $t \in \mathbf{R}$,

$$
|\phi(i t, h)|=1 .
$$

(b) For all $z \in \mathbb{C}$,

$$
\phi(-z, h)=\frac{1}{\phi(z, h)} .
$$

(c) For all $x \in \mathbf{R}^{+}$,

$$
|\phi(x, h)| \leq\left(k^{\prime}\right)^{1 / 2} .
$$

(d) For $j=0, \pm 1, \pm 2, \ldots$,

$$
\phi^{\prime}\left(e^{j h}, h\right)=\frac{\left(k^{\prime}\right)^{1 / 2} K}{\pi}(-1)^{j} e^{-j h} .
$$

(e) If $\theta \in \mathbf{R}$ and $j=0, \pm 1, \pm 2, \ldots$, then

$$
\left(k^{\prime}\right)^{1 / 2} \leq\left|\phi\left(e^{(j+1 / 2) h} e^{i \theta}, h\right)\right| \leq\left(k^{\prime}\right)^{-1 / 2} .
$$

Proof. (a) The relation (2.5) follows at once from (2.1), since each of the factors $\left(i t-e^{j h}\right) /\left(i t+e^{j h}\right)$ has modulus 1 for all $t \in \mathbf{R}$.

(b) The relation (2.6) follows from (2.1). 
(c) The relation (2.7) is a consequence of the identity (2.4) and the fact that $\operatorname{sn}\left(u, k^{\prime}\right)$ maps $\mathbf{R}$ onto $[-1,1]$.

(d) Differentiating the right-hand side of (2.4) and using the formula [28, Section 16.16.1]

$$
\frac{d}{d u} \operatorname{sn}\left(u ; k^{\prime}\right)=\operatorname{cn}\left(u ; k^{\prime}\right) \operatorname{dn}\left(u ; k^{\prime}\right)
$$

we get (2.8).

(e) Using [28, Eq. 16.21.2], we have

$$
\phi\left(e^{(j+1 / 2) h} e^{i \theta}, h\right)=(-1)^{j}\left(k^{\prime}\right)^{1 / 2} / \operatorname{dn}\left(\frac{K \theta}{\pi} ; k\right) .
$$

But for $u \in \mathbb{R}, k^{\prime} \leq \operatorname{dn}(u ; k) \leq 1$. Hence (2.9) follows.

LEMMA 2.2. If $h=2 \log (1 / q)$, then:

(a) The bound

$$
k^{\prime} \leq 4 e^{-\pi^{2} / h}
$$

is valid for all $h>0$.

(b) $A s h \rightarrow 0$,

$$
k^{\prime}=e^{-\pi^{2} / h}\left[4+O\left(e^{-2 \pi^{2} / h}\right)\right] .
$$

(c) $A s h \rightarrow 0$,

$$
\frac{K\left(k^{\prime}\right)^{1 / 2}}{\pi}=\left(\frac{2 \pi}{h}\right) e^{-\pi^{2} /(2 h)}\left[1+O\left(e^{-2 \pi^{2} / h}\right)\right] .
$$

Proof. The inequality (2.12) was verified in [40]. The relation (2.13) results upon taking the ratio of the two equations $[28,16.38 .5]$ and $[28,16.38 .7]$, which yield the upper and lower estimates

$$
\frac{2 e^{-\pi^{2} /(2 h)}}{1+2 e^{-2 \pi^{2} / h}} \leq\left(k^{\prime}\right)^{1 / 2} \leq \frac{2 e^{-\pi^{2} /(2 h)}\left(1+e^{-4 \pi^{2} / h}\right)}{1+e^{-2 \pi^{2} / h}} .
$$

Finally, writing $K=\left\{K /\left(\pi K^{\prime}\right)\right\} \pi K^{\prime}$, and using [28, Eq. 16.38.6] as well as (2.13) and the identity $q^{\prime}=e^{-2 \pi^{2} / h}$, we get $(2.14)$.

Proof of Theorem 1.1. Let $\Omega_{+}$denote the right half plane, i.e.,

$$
\Omega_{+}=\{z \in \mathbb{C}: \operatorname{Re} z>0\},
$$

and for $h>0$ and $N$ a positive integer, let $A_{N}$ denote the region

$$
A_{N}=\left\{z \in \mathbb{C}: \operatorname{Re} z>0, e^{-(N+1 / 2) h}<|z|<e^{(N+1 / 2) h}\right\} .
$$

Let $s \in A_{N}$, and set

$$
\varepsilon_{N}(g, h, s)=\frac{\phi(s, h)}{2 \pi i} \int_{\partial A_{N}} \frac{g(z) d z}{(z-s) \phi(z, h)} .
$$

Using Lemma 2.1(d), we can evaluate the contour integral in (2.18) by summing the residues to get

$$
\varepsilon_{N}(g, h, s)=g(s)-\frac{\pi \phi(s, h)}{\left(k^{\prime}\right)^{1 / 2} K} \sum_{n=-N}^{N} \frac{(-1)^{n} e^{n h} g\left(e^{n h}\right)}{s-e^{n h}} .
$$


Now given $G$ defined on $\Omega_{+}$such that

$$
\|G\|=\left(\int_{\mathbf{R}}\left|G\left(i y+0^{+}\right)\right|^{2} d y\right)^{1 / 2}<\infty
$$

the function $G^{+}$defined for $s \in \Omega_{+}$by

$$
G^{+}(s)=\frac{1}{2 \pi i} \int_{\mathbf{R}} \frac{G\left(i u+0^{+}\right) d u}{i u-s}
$$

satisfies the relation

$$
\int_{\mathbf{R}}\left|G^{+}(c+i u)\right|^{2} d u \leq\|G\|^{2}
$$

for every $c>0$. Consequently, since $|\phi(i u, h)|=1$ for $u \in \mathbb{R}$, the function $w$ defined for $s \in \Omega_{+}$by

$$
w(s)=\lim _{\varepsilon \rightarrow 0^{+}} \int_{-i \infty}^{i \infty} \frac{g(z+\varepsilon) d z}{\phi(z, h)(z-s)}
$$

satisfies the relation

$$
\int_{\mathbf{R}}|w(c+i u)|^{2} d u \leq\|g\|^{2}
$$

for every $c>0$.

It is then easily shown, using the results of Lemmas 2.1 and 2.2 , that for $s \in \Omega_{+}$, the limits

$$
\varepsilon(g, h, s)=\lim _{N \rightarrow \infty} \varepsilon_{N}(g, h, s)=\lim _{\eta \rightarrow 0^{+}} \frac{1}{2 \pi i} \int_{-i \infty}^{i \infty} \frac{\phi(s, h) g(z+\eta) d z}{\phi(z, h)(z-s)}
$$

exist; indeed, upon applying Schwarz's inequality to the integral on the right-hand side of $(2.25)$, we find that

$$
|\varepsilon(g, h, s)| \leq \frac{|\phi(s, h)|\|g\|}{2 \operatorname{Re} s} .
$$

In particular, if $s \in \mathbb{R}^{+}$, then by Lemma $2.1(\mathrm{c})$ we have $|\phi(s, h)| \leq\left(k^{\prime}\right)^{1 / 2}$, which shows by (2.12) that $\varepsilon(g, h, s) \rightarrow 0$ as $h \rightarrow 0$ for all $s \in \mathbb{R}^{+}$.

The error $\varepsilon(g, h, s)$ is also given by

$$
\varepsilon(g, h, s)=g(s)-\frac{\pi}{\left(k^{\prime}\right)^{1 / 2} K} \sum_{n=-\infty}^{\infty} \frac{(-1)^{n} e^{n h} g\left(e^{n h}\right) \phi(s, h)}{s-e^{n h}} .
$$

We shall now show that for every $c>0$,

$$
\int_{\mathbf{R}}|\varepsilon(g, h, c+i u)|^{2} d u \rightarrow 0
$$

as $h \rightarrow 0$.

We observe that $\log |\phi(s, h)|$ is harmonic in the region $0<\theta<\pi / 2$, where $\theta=\arg s$. Moreover, by Lemma 2.1,

$$
\begin{aligned}
& \lim _{\theta \rightarrow 0^{+}} \log \left|\phi\left(|s| e^{i \theta}, h\right)\right| \leq \frac{1}{2} \log k^{\prime}, \\
& \lim _{\theta \rightarrow \pi / 2} \log \left|\phi\left(|s| e^{i \theta}, h\right)\right|=0 .
\end{aligned}
$$


Consequently, for $\theta$ in the range $0<\theta<\pi / 2$, we have

$$
\log \left|\phi\left(|s| e^{i \theta}, h\right)\right| \leq \frac{1}{2}\left(1-\frac{2 \theta}{\pi}\right) \log k^{\prime} .
$$

That is,

$$
\left|\phi\left(|s| e^{i \theta}, h\right)\right| \leq\left(k^{\prime}\right)^{1 / 2(1-2 \theta / \pi)} .
$$

Hence, if $s=c+i u$, where $c>0$ and $u \in \mathbb{R}$, then

$$
|\phi(c+i u, h)| \leq\left(k^{\prime}\right)^{(1 / \pi)\left|\tan ^{-1}(c / u)\right|} .
$$

In view of (2.18), (2.23), (2.24) and the fact that $|\phi(s, h)| \leq 1$ if $s \in \Omega_{+}$it follows that, given any $\eta>0, c>0$, there exists a $T>0$, such that

$$
\frac{1}{2 \pi} \int_{|u|>T}|\varepsilon(g, h, c+i u)|^{2} d u<\frac{\eta / 2}{2 \pi}\|g\|^{2} .
$$

Also, by (2.18), (2.23), (2.24), (2.25), and (2.32),

$$
\begin{aligned}
& \frac{1}{2 \pi} \int_{-T}^{T}|\varepsilon(g, h, c+i u)|^{2} d u \\
& \quad \leq \max _{|u| \leq T}|\phi(c+i u, h)|^{2} \frac{1}{2 \pi} \int_{-T}^{T}|w(c+i u)|^{2} d u \\
& \quad \leq \frac{\left(k^{\prime}\right)^{(2 / \pi) \tan ^{-1}(c / T)}}{2 \pi}\|g\|^{2} .
\end{aligned}
$$

Hence, by combining $(2.33),(2.34)$ and $(2.12)$, we see that if $\left(k^{\prime}\right)^{(2 / \pi) \tan ^{-1}(c / T)} \leq$ $\eta / 2$, then

$$
\frac{1}{2 \pi} \int_{\mathbf{R}}|\varepsilon(g, h, c+i u)|^{2} d u<\frac{\eta\|g\|^{2}}{2 \pi}
$$

From the identity

$$
\begin{aligned}
\delta(g, h, t) & \equiv \frac{1}{2 \pi i} \int_{c-i \infty}^{c+i \infty} e^{s t} \varepsilon(g, h, s) d s \\
& =f(t)-\frac{\pi^{2}}{k^{\prime} K^{2}} \sum_{m, n=-\infty}^{\infty} \frac{\left(-e^{h}\right)^{m+n} \exp \left(-t e^{m h}\right) g\left(e^{n h}\right)}{e^{m h}+e^{n h}}
\end{aligned}
$$

it now follows from (2.35) and Parseval's theorem that

$$
\int_{0}^{\infty} e^{-2 c t}|\delta(g, h, t)|^{2} d t=\frac{1}{2 \pi} \int_{\mathbf{R}}|\varepsilon(g, h, c+i u)|^{2} d u<\eta \frac{\|g\|^{2}}{2 \pi} .
$$

This completes the proof of Theorem 1.1.

3. Proofs of Theorems for Method II. Let us note that the operator $\mathscr{L}$ defined by (1.1) is selfadjoint, so that $\mathscr{L}=\mathscr{L}^{*}$. It was known that 0 is in the spectrum of $\mathscr{L}$ as an operator on $L^{2}\left(\mathbb{R}^{+}\right)$to $L^{2}\left(\mathbb{R}^{+}\right)$(see, e.g., [14]). Hence, $\mathscr{L}$ is not continuously invertible, i.e., finding the inverse of a Laplace transform is an ill-posed problem. It is clear, however, that for each $\beta>0$, the operator $\beta+\mathscr{L}^{*} \mathscr{L}$ is continuously invertible on $L^{2}\left(\mathbb{R}^{+}\right)$. 
Proof of Theorem 2.1. Let us form a spectral decomposition of $\mathscr{L}$ in the form [32, pp. 277-280]

$$
\mathscr{L}=\mathscr{L}_{+}-\mathscr{L}_{-}
$$

and let us set

$$
|\mathscr{L}|=\mathscr{L}_{+}+\mathscr{L}_{-}
$$

Now, from (1.10), one gets

$$
\beta\left(f_{\beta}-f\right)+\mathscr{L}^{*} \mathscr{L}\left(f_{\beta}-f\right)=\mathscr{L}^{*} g-\mathscr{L}^{*} \mathscr{L} f-\beta f
$$

and hence

$$
f_{\beta}-f=\left(\beta+\mathscr{L}^{*} \mathscr{L}\right)^{-1} \mathscr{L}^{*}(g-\mathscr{L} f)-\left(\beta+\mathscr{L}^{*} \mathscr{L}\right)^{-1} \beta f .
$$

We note that $\beta+\mathscr{L}^{*} \mathscr{L}$ commutes with both $\mathscr{L}$ and $|\mathscr{L}|$, and hence so does $\left(\beta+\mathscr{L}^{*} \mathscr{L}\right)^{-1}$. Also, $\left(\beta+\mathscr{L}^{*} \mathscr{L}\right)^{-1}$ is a positive and symmetric operator. Moreover, $|\mathscr{L}| \geq \pm \mathscr{L}$, and hence $\left(\beta+\mathscr{L}^{*} \mathscr{L}\right)^{-1}|\mathscr{L}| \geq \pm\left(\beta+\mathscr{L}^{*} \mathscr{L}\right)^{-1} \mathscr{L}$. Thus, for any $x \in L^{2}\left(\mathbf{R}^{+}\right)$,

$$
\left(\left(\beta+\mathscr{L}^{*} \mathscr{L}\right)^{-1}|\mathscr{L}| x, x\right) \geq\left|\left(\left(\beta+\mathscr{L}^{*} \mathscr{L}\right)^{-1} \mathscr{L} x, x\right)\right| .
$$

Recalling that for a symmetric operator $A$, we have $\|A\|=\sup _{\|x\|=1}|(A x, x)|$, it thus follows that

$$
\left\|\left(\beta+\mathscr{L}^{*} \mathscr{L}\right)^{-1}|\mathscr{L}|\right\| \geq\left\|\left(\beta+\mathscr{L}^{*} \mathscr{L}\right)^{-1} \mathscr{L}\right\| .
$$

Now, we have $\mathscr{L}^{*} \mathscr{L}=\mathscr{L}^{2}=|\mathscr{L}|^{2}$, and so for any $x \in L^{2}\left(\mathbb{R}^{+}\right)$,

$$
0 \leq\left((\sqrt{\beta}-|\mathscr{L}|)^{2} x, x\right)=\left(\left(\beta+\mathscr{L}^{*} \mathscr{L}-2 \sqrt{\beta}|\mathscr{L}|\right) x, x\right) .
$$

That is,

$$
\beta+\mathscr{L}^{*} \mathscr{L}-2 \sqrt{\beta}|\mathscr{L}| \geq 0 \text {. }
$$

Hence,

$$
\left(\beta+\mathscr{L}^{*} \mathscr{L}\right)^{-1}\left(\beta+\mathscr{L}^{*} \mathscr{L}-2 \sqrt{\beta}|\mathscr{L}|\right) \geq 0
$$

which implies that

$$
\left\|\left(\beta+\mathscr{L}^{*} \mathscr{L}\right)^{-1}|\mathscr{L}|\right\| \leq 1 / 2 \sqrt{\beta} .
$$

Thus, if $\beta=\varepsilon$, then, since $\|g-\mathscr{L} f\| \leq \varepsilon$,

$$
\left\|\left(\beta+\mathscr{L}^{*} \mathscr{L}\right)^{-1} \mathscr{L}^{*}(g-\mathscr{L} f)\right\| \leq \frac{\varepsilon}{2 \sqrt{\beta}}=\frac{\sqrt{\varepsilon}}{2} .
$$

Also, since by assumption $f=\mathscr{L} u, u \in L^{2}\left(\mathbb{R}^{+}\right)$, we have

$$
\left\|\left(\beta+\mathscr{L}^{*} \mathscr{L}\right)^{-1} f\right\|=\left\|\left(\beta+\mathscr{L}^{*} \mathscr{L}\right)^{-1} \mathscr{L} u\right\| \leq \frac{E}{2 \sqrt{\beta}}=\frac{E}{2 \sqrt{\varepsilon}}
$$

so that

$$
\left\|\left(\beta+\mathscr{L}^{*} \mathscr{L}\right)^{-1} \beta f\right\| \leq \frac{E \sqrt{\varepsilon}}{2} .
$$

Combining (3.12) and (3.13) with (3.4) we get (1.12).

Proof of Theorem 2.3. This is a direct consequence of Theorems 2.1 and 2.4 of Ikebe [19]. 
4. Proofs of Theorems for Method III. In this section we shall prove Theorems 3.2, 3.3, 3.4, 3.6 and Corollary 3.7, which were stated in the introductory section.

Proof of Theorem 3.2. Using Eqs. (1.26) and (1.28), we get

$$
\beta\left(\hat{F}_{\beta}-\hat{F}\right)+|\hat{H}|^{2}\left(\hat{F}_{\beta}-\hat{F}\right)=\overline{\hat{H}}\left(\hat{G}-\hat{G}_{0}\right)-\beta \hat{F},
$$

This is, upon multiplying both sides of this equation by $\overline{\left(\hat{F}_{\beta}-\hat{F}\right)}$ and integrating over $\mathbf{R}$,

$$
\left\|\left(\beta+|\hat{H}|^{2}\right)\left|\hat{F}_{\beta}-\hat{F}\right|^{2}\right\|_{1} \leq\left\|\overline{\hat{H}}\left(\hat{G}-\hat{G}_{0}\right) \overline{\left(\hat{F}_{\beta}-\hat{F}\right)}\right\|_{1}+\beta\left\|\hat{\bar{F}\left(\hat{F}_{\beta}-\hat{F}\right)}\right\|_{1} .
$$

But

$$
\hat{H}(x)=\int_{\mathbf{R}} e^{i x u} \exp \left[-e^{-u}\right] e^{-u} d u=\Gamma(1-i x),
$$

where $\Gamma(\cdot)$ denotes the usual Gamma function, and therefore [28, Eq. 6.1.31]

$$
|\hat{H}(x)|^{2}=\hat{H}(x) \overline{\hat{H}(x)}=\frac{\pi x}{\sinh (\pi x)} .
$$

Hence, $\sup _{x \in \mathbf{R}}|\hat{H}(x)|=1$, and so (4.2) yields

$$
\left\|\hat{F}_{\beta}-\hat{F}\right\|_{2}^{2} \leq \frac{1}{\beta}\left\|\hat{G}-\hat{G}_{0}\right\|_{2}\left\|\hat{F}_{\beta}-\hat{F}\right\|_{2}+\|\hat{F}\|_{2}\left\|\hat{F}_{\beta}-\hat{F}\right\|_{2} .
$$

Using (1.27) in (4.5), we get (1.29).

Proof of Theorem 3.3. Let us multiply Eq. (4.1) by $x^{2 s} \overline{\left(\hat{F}_{\beta}-\hat{F}\right)}$ and then integrate over $\mathbf{R}$, to get

$$
\begin{aligned}
\beta \int_{\mathbf{R}} x^{2 s}\left|\hat{F}_{\beta}(x)-\hat{F}(x)\right|^{2} d x+\int_{\mathbf{R}}|\hat{H}(x)|^{2} x^{2 s}\left|F_{\beta}(x)-F(x)\right|^{2} d x \\
=\int_{\mathbf{R}} \overline{\hat{H}}(x) x^{s}\left[\hat{G}(x)-\hat{G}_{0}(x)\right] x^{s} \overline{\left[F_{\beta}(x)-\hat{F}(x)\right]} d x \\
\quad-\beta \int_{\mathbf{R}} x^{s} \hat{F}(x) x^{s} \overline{\left[\hat{F}_{\beta}(x)-\hat{F}(x)\right]} d x .
\end{aligned}
$$

Now using (1.27), (1.30), (1.31) and (4.6), we get

$$
\begin{aligned}
\int_{\mathbf{R}}[\beta & \left.+|\hat{H}(x)|^{2}\right] x^{2 s}\left|\hat{F}_{\beta}(x)-\hat{F}(x)\right|^{2} d x \\
& \leq\left(\alpha_{s} \varepsilon+\beta E_{s}\right)\left(\int_{\mathbf{R}} x^{2 s}\left|\hat{F}_{\beta}(x)-\hat{F}(x)\right|^{2} d x\right)^{1 / 2} .
\end{aligned}
$$

Hence, (4.7) yields

$$
\left(\int_{\mathbf{R}} x^{2 s}\left|\hat{F}_{\beta}(x)-\hat{F}(x)\right|^{2} d x\right)^{1 / 2} \leq \frac{\alpha_{s} \varepsilon+\beta E_{s}}{\beta}=\alpha_{s} E_{0}+E_{s}
$$

Next, from (4.2) and the definition of $\beta$ in Theorem 3.2, we have

$$
\begin{aligned}
\int_{\mathbf{R}}|\hat{H}(x)|^{2}\left|\hat{F}_{\beta}(x)-\hat{F}(x)\right|^{2} d x \\
\leq \int_{\mathbf{R}}|\overline{\hat{H}(x)}|\left|\hat{G}(x)-\hat{G}_{0}(x)\right|\left|\overline{\hat{F}_{\beta}(x)-\hat{F}(x)}\right| d x \\
\quad+\beta \int_{\mathbf{R}}|\hat{F}(x)|\left|\overline{\hat{F}_{\beta}(x)-\hat{F}(x)}\right| d x \leq 4 E_{0} \varepsilon .
\end{aligned}
$$


Let $x_{s}>0$ be determined by (1.31). Then, since $|\hat{H}(x)|^{2}$ is decreasing and $x^{2 s}$ is increasing as $|x|$ increases, we have

$$
\begin{aligned}
\int_{|x|<x_{s}}\left|\hat{F}_{\beta}(x)-\hat{F}(x)\right|^{2} d x & \leq \int_{|x|<x_{s}}\left|\frac{\hat{H}(x)}{\hat{H}\left(x_{s}\right)}\right|^{2}\left|\hat{F}_{\beta}(x)-\hat{F}(x)\right|^{2} d x \\
& \leq \frac{1}{\left|\hat{H}\left(x_{s}\right)\right|^{2}} \cdot 4 E_{0} \varepsilon=\frac{\alpha_{s} E_{0}+E_{s}}{x_{0}^{2 s}} .
\end{aligned}
$$

Similarly, we have

$$
\begin{aligned}
\int_{|x|>x_{s}}\left|\hat{F}_{\beta}(x)-\hat{F}(x)\right|^{2} d x & \leq \frac{1}{x_{s}^{2 s}} \int_{|x|>x_{s}} x^{2 s}\left|\hat{F}_{\beta}(x)-\hat{F}(x)\right|^{2} d x \\
& \leq \frac{\alpha_{s} E_{0}+E_{s}}{x_{s}^{2 s}} .
\end{aligned}
$$

By adding (4.10) and (4.11), we get (1.32).

We remark that an estimate of $\alpha_{s}$ defined in (1.31) is

$$
\alpha_{s} \cong(2 \pi)^{1 / 2}\left(\frac{2 s+1}{e}\right)^{s+1 / 2} .
$$

This estimate is obtained upon replacing $1 / \sinh (\pi x)$ by $2 / e^{|\pi x|}$ over $\mathbf{R}$.

The estimate (1.33) is obtained similarly, by first replacing $\sinh \pi x$ by $\frac{1}{2} e^{|\pi x|}$.

Proof of Theorem 3.4. Let $\xi>0$ be defined by the equation

$$
\pi \xi e^{2 \xi d}=\frac{\pi \xi}{\sinh \pi \xi} \frac{E_{d}}{4 E_{0} \varepsilon} .
$$

Then,

$$
\begin{aligned}
& \pi \xi e^{2 \xi d} \int_{|x|>\xi}\left|\hat{F}_{\beta}(x)-\hat{F}(x)\right|^{2} d x \\
& \quad \leq \int_{|x|>\xi} \pi|x| e^{2 d|x|}\left|\hat{F}_{\beta}(x)-\hat{F}(x)\right|^{2} d x \leq E_{d} .
\end{aligned}
$$

Similarly, by (4.13) and (4.9),

$$
\begin{aligned}
\frac{4 E_{0} \varepsilon}{E_{d}} & \pi \xi e^{2 \xi d} \int_{|x|<\xi}\left|\hat{F}_{\beta}(x)-\hat{F}(x)\right|^{2} d x \\
& =\frac{\pi \xi}{\sinh \pi \xi} \int_{|x|<\xi}\left|\hat{F}_{\beta}(x)-\hat{F}(x)\right|^{2} d x \\
& \leq \int_{|x|<\xi}|\hat{H}(x)|^{2}\left|\hat{F}_{\beta}(x)-\hat{F}(x)\right|^{2} d x \leq 4 E_{0} \varepsilon
\end{aligned}
$$

By combining (4.14) and (4.15), we get

$$
\left\|\hat{F}_{\beta}-\hat{F}\right\|^{2} \leq \frac{2 E_{d}}{\pi \xi e^{2 \xi d}} .
$$

Starting with (4.13), let us estimate $\xi$ by $\xi_{0}$, where

$$
e^{(2 d+\pi) \xi_{0}}=\frac{E_{d}}{2 E_{0} \varepsilon} .
$$

That is, by comparing the right-hand sides of (4.13) and (4.17), we have

$$
\pi \xi_{0} \leq \pi \xi \Rightarrow e^{2 \xi_{0} d} \leq e^{2 \xi d}
$$


so that

$$
\frac{2 E_{d}}{\pi \xi e^{2 \xi d}}<\frac{2 E_{d}}{\pi \xi_{0} e^{2 \xi_{0} d}}
$$

Upon solving (4.17) for $\xi_{0}$ and then substituting into (4.16), we get (1.35).

Proof of Theorem 3.6. The identity

$$
\hat{F}_{\beta}-\hat{F}_{\beta, h}=\frac{\overline{\hat{H}} \hat{G}-\overline{\hat{H}}_{h} \hat{G}_{h}}{\beta+|\hat{H}|^{2}}=\frac{\overline{\hat{H}}\left(\hat{G}-\hat{G}_{h}\right)+\left(\overline{\hat{H}}-\overline{\hat{H}}_{h}\right) \hat{G}_{h}}{\beta+|\hat{H}|^{2}}
$$

shows that the error in $\hat{F}_{\beta}$ is a sum of errors in $\hat{G}$ and $\hat{H}$. If Assumptions 2.2 and 3.5 are satisfied, then by [39], [40] both of these errors are $O\left(N^{1 / 2} e^{-(\pi d \alpha N)^{1 / 2}}\right)$, and hence so is the error in $\hat{F}_{\beta}-\hat{F}_{\beta, h}$, as well as $F_{\beta}-F_{\beta, h}$, whose Fourier transform over $\mathbf{R}$ is $\hat{F}_{\beta}-\hat{F}_{\beta, h}$. The inequality (1.40) thus follows.

Proof of Corollary 3.7. The proof of Corollary 3.7 is the same as the proof of Theorem 3.6, upon noting that analyticity and boundedness of $\hat{F}$ in $S_{\alpha}$ implies that the inequalities (1.14) are satisfied.

5. Examples. Each of the functions $g$ which are given (along with their inverse Laplace transforms $f$ ) in Table 5.1 were tested, yielding results of accuracy similar to that indicated in Figures 1.4.1 and 1.4.2, with all inversion methods of this paper.

TABLE 5.1

Test functions

\begin{tabular}{c|l}
\hline$g(s)$ & \multicolumn{1}{c}{$f(t)$} \\
\hline$(s+1)^{-2}$ & $t e^{-t}$ \\
$\left(s^{2}+2 s++2\right)^{-1}$ & $e^{-t} \sin (t)$ \\
$\Gamma(4 / 3)(s+4 / 3)^{-7 / 3}$ & $t^{4 / 3} e^{-4 t / 3}$ \\
$\Gamma(5 / 2)(s+1)^{-5 / 2}[\psi(5 / 2)-\log (s+1)]$ & $t^{3 / 2} e^{-t} \log (t)$ \\
\hline
\end{tabular}

The function $f(t)=\operatorname{sn}(t ; k) e^{-t}$ is analytic and bounded in $B_{d}(0<d<\pi)$, but not in $S_{d}$ for any $d>0$, and for this reason Method II corresponding to $B_{d}$ was much more accurate than Method II corresponding to $S_{d}$, when each was applied to the inversion of the Laplace transform of $f$, which we were able to express in this case only as an infinite series.

Finally, we also attempted to invert the Laplace transform $g(s)=\left(s^{2}+1\right)^{-1}$ of $f(t)=\sin t$. In this case, $f$ does not belong to $L^{2}\left(\mathbb{R}^{+}\right)$, and Sinc interpolation of $f$ on $\mathbb{R}^{+}$does not work, since for accurate Sinc interpolation on $\mathbb{R}^{+}$we must have $f(t)$ approach zero both as $t \rightarrow 0$ and also as $t \rightarrow \infty$. Hence, although Method I worked for $g(s)=\left(s^{2}+1\right)^{-1}$, it converged very slowly. Methods II and III gave accurate results only near $t=0$.

Copies of FORTRAN programs for Methods I, II, and III may be obtained from the third author. 
Acknowledgment. D. D. Ang wishes to acknowledge support from the University of Utah, where he was a visitor while this research was carried out. F. Stenger wishes to thank the U.S. Army, Contract No. DAAL 03-87-K-0008 for research support. The authors are grateful to the referee and managing editor for valuable criticisms.

Department of Mathematics

Dai Hoc Tong Hop

Ho Chi Minh City University

Viet Nam

Department of Mathematics

Montana State University

Bozeman, Montana 59715

Department of Mathematics

University of Utah

Salt Lake City, Utah 84112

E-mail: stenger@science.utah.edu

1. R. Bellman, R. E. Kalaba \& J. A. Lockett, Numerical Inversion of the Laplace Transform, American Elsevier, New York, 1966.

2. B. S. BERGER, "Inversion of the $n$-dimensional Laplace transform," Math. Comp., v. 20, 1966 , pp. 418-421.

3. B. S. BERGER, "The inversion of the Laplace transform with application to the vibrations of continuous elastic bodies," J. Appl. Mech., v. 35, 1968, pp. 837-839.

4. B. S. BERGER \& S. DUANGUDOM, "A technique for increasing the accuracy of the numerical inversion of the Laplace transform with applications," J. Appl. Mech., v. 40, 1973, pp. 1110-1112.

5. C. BREZINSKI \& J. VAN ISEGHEM, "Padé-type approximants and linear functional transformations," in Rational Approximation and Interpolation, Lecture Notes in Math., vol. 1105, SpringerVerlag, Berlin and New York, 1984, pp. 100-108.

6. J. W. COOLEY, P. A. W. LEWIS \& P. D. WELCH, "The fast Fourier transform algorithm: Programming considerations in the calculation of sine, cosine, and Laplace transforms," J. Sound Vibration, v. 12, 1970, pp. 315-337.

7. J. W. COOLEY \& J. W. TUKEY, "An algorithm for the machine calculation of complex Fourier series," Math. Comp., v. 19, 1965, pp. 297-301.

8. K. S. CRUMP, "Numerical inversion of Laplace transforms using Fourier series approximation," J. Assoc. Comput. Mach., v. 23, 1976, pp. 89-96.

9. A. R. DAviES, "On the maximum likelihood regularization of Fredholm convolution equations of the first kind," in Treatment of Integral Equations by Numerical Methods (C. T. H. Baker and G. F. Miller, eds.), Academic Press, New York, 1982, pp. 95-105.

10. B. DAvies \& B. MARTin, "Numerical inversion of the Laplace transform: A survey and comparison of methods," J. Comput. Phys., v. 33, 1979, pp. 1-32.

11. H. DUBNER \& J. ABATE, "Numerical inversion of Laplace transforms by relating them to the finite Fourier cosine transform," J. Assoc. Comput. Mach., v. 15, 1968, pp. 115-123.

12. A. ERDÉLYI, "Inversion formula for the Laplace transform," Philos. Mag., v. 34, 1943, pp. 533-537.

13. W. Feller, An Introduction to Probability Theory and its Applications, Vol. 2, Wiley, New York, 1971.

14. D. S. Gilliam, J. R. SChulenberger \& J. R. Lund, "Spectral representation of the Laplace and Stieltjes transform," in Computational and Combinatorial Methods in Systems Theory (C. P. Byrne and A. Lindquist, eds.), Elsevier Science, North-Holland, 1986, pp. 69-74.

15. R. R. Goldberg \& R. S. VARGA, "Moebius inversion of Fourier transforms," Duke Math. $J .$, v. 23,1956 , pp. $553-559$.

16. S. A. GUSTAFSON \& G. DAHLQUist, "On the computation of slowly convergent Fourier integrals," in Methoden und Verfahren der Mathematischen Physik, no. 6, Bibliographisches Institut Ag Mannheim, Wien and Zürich, 1972, pp. 93-112.

17. P. Henrici, Applied Computational and Complex Analysis, Vol. 2, Wiley, New York, 1977. 
18. F. R. DeHoog, J. H. KNIGHT \& A. N. StokeS, "An improved method for numerical inversion of Laplace transforms," SIAM J. Sci. Statist. Comput., v. 3, 1982, pp. 357-366.

19. Y. IKEBE, "Galerkin method for the numerical solution of Fredholm integral equations of the second kind," SIAM Rev., v. 14, 1972, pp. 465-491.

20. R. E. JONES, Solving Linear Algebraic Systems Arising in the Solution of Integral Equations of the First Kind, Ph.D. Dissertation, University of New Mexico, 1985.

21. I. M. LONGMAN, "Note on a method for computing infinite integrals of oscillatory functions," Proc. Cambridge Philos. Soc., v. 52, 1956, pp. 764-768.

22. Y. L. LUKE, "On the computation of oscillatory integrals," Proc. Cambridge Philos. Soc., v. 50,1954, pp. $269-277$.

23. J. ROSS MACDONALD, "Accelerated convergence, divergence, iteration, extrapolation, and curve fitting," J. Appl. Phys., v. 35, 1964, pp. 3034-3041.

24. M. J. MARSDEN \& G. D. TAYLOR, "Numerical evaluation of Fourier integrals," in $\mathrm{Nu}$ merische Methoden der Approximationstheorie, Band 1 (L. Collatz and G. Meinardus, eds.), ISNM 16, Birkhäuser-Verlag, Basel, 1972, pp. 61-76.

25. J. T. MARTI, "On a regularization method for Fredholm equations of the first kind using Sobolev spaces," in Treatment of Integral Equations by Numerical Methods (C. T. H. Baker and G. F. Miller, eds.), Academic Press, New York, 1982, pp. 59-66.

26. P. DEMOtToni \& G. TAlenti, "Stabilization and error bounds for the inverse Laplace transform," Numer. Funct. Anal. Optim., v. 3, 1981, pp. 265-283.

27. M. Z. NASHED \& G. WAHBA, "Some exponentially decreasing error bounds for a numerical inversion of the Laplace transform," J. Math. Anal. Appl., v. 52, 1975, pp. 660-668.

28. National Bureau of Standards, Handbook of Mathematical Functions, vol. 55, Applied Math. Series, Pitman, Boston, 1964.

29. A. Papoulis, "A new method of inversion of the Laplace transform," Quart. Appl. Math., v. 14, 1956, pp. 405-414.

30. R. PIESSENS, "Numerical inversion of the Laplace transform," IEEE Trans. Automat. Control, v. AC-14, 1969, pp. 299-301.

31. E. L. Post, "Generalized differentiation," Trans. Amer. Math. Soc., v. 32, 1930, pp. 723-781.

32. F. RIESZ \& B. SZ-NAGY, Functional Analysis, Ungar, New York, 1955.

33. H. E. SALZER, "Tables for the numerical calculation of inverse Laplace transforms," J. Math. Phys., v. 37, 1958, pp. 89-109.

34. H. E. SALZER, "Additional formulas and tables for orthogonal polynomials originating from inversion integrals," J. Math. Phys., v. 40, 1961, pp. 72-86.

35. R. A. SCHAPERY, Approximate Methods of Transform Inversion for Viscoelastic Stress Analysis, Proc. 4th U.S. Nat. Congr. Appl. Mech. ASME, no. 2, 1962, pp. 1075-1085.

36. C. J. ShiRTLiffe \& D. G. STEPhENSON, "A computer oriented adaption of Salzer's method for inverting Laplace transforms," J. Math. Phys., v. 40, 1961, pp. 135-141.

37. K. SinghaL \& J. VLACH, "Computation of time domain response by numerical inversion of the Laplace transform," J. Franklin Inst., v. 299, 1975, pp. 109-126.

38. M. SilVERBERG, "Improving the efficiency of Laplace transform inversion for network analysis," Electron. Lett., v. 6, 1970, pp. 105-106.

39. F. STENGER, "Numerical methods based on the Whittaker cardinal, or Sinc functions," SIAM Rev., v. 23, 1981, pp. 165-224.

40. F. STENGER, "Explicit, nearly optimal, linear rational approximation with preassigned poles," Math. Comp., v. 47, 1986, pp. 225-252.

41. A. N. Tikhonov \& V. Y. ARsenin, Solution of Ill-Posed Problems, Winston, Washington, 1977.

42. M. TRUMMER, "A method of solving ill-posed linear operator equations," SIAM J. Numer. Anal., v. 21, 1984, pp. 729-737.

43. D. V. WIdDER, The Laplace Transform, Princeton Univ. Press, Princeton, N.J., 1936.

44. V. ZAKIAN, "Numerical inversion of Laplace transform," Electron. Lett., v. 5, 1969, pp. $120-121$.

45. V. ZAKIAN, "Optimization of numerical inversion of Laplace transform," Electron. Lett., v. 6,1970, pp. $677-679$.

46. V. ZAKIAN \& D. R. GANNON, "Least squares optimization of numerical inversion of Laplace transform," Electron. Lett., v. 7, 1971, pp. 70-71. 